\title{
Sintilimab as salvage treatment in an HIV patient with relapsed/ refractory Hodgkin: a case report
}

\author{
Yang Shi", Qiying Li", Wenjun Zhang, Yingyu Nan, Tao Yang, Xiping Liang, Chunyan Xiao, Bingling Guo, \\ Ying Xiang \\ Department of Hemo-oncolgy, Chongqing University Cancer Hospital, Chongqing, China \\ \#These authors contributed equally to this work. \\ Correspondence to: Ying Xiang. Department of Hemo-oncolgy, Chongqing University Cancer Hospital, Chongqing, China. Email: xiangying0331@sina.com.
}

\begin{abstract}
The prognosis of relapsed/refractory classical Hodgkin lymphoma companied with Human immunodeficiency virus (R/R HIV-cHL) is poor due to insufficient effective treatments. Nowadays, immune checkpoint blockade is an important new treatment option for patients with relapsed/refractory classical Hodgkin lymphoma (cHL), but rare cases have been reported in R/R HIV-cHL. We present a case of R/R HIV-cHL young patient, who has been successfully treated with sintilimab without significant side effects. In May 2018, we received an Hodgkin lymphoma companied with Human immunodeficiency virus (HIVcHL) patient. At first, we gave him ABVD regime chemotherapy. In April 2019, after 6 cycles of ABVD and radiation, we evaluated the effect of treatment and found that the disease actually progressed. The patient refused auto stem cell transplant, so the second line GDP regime chemotherapy was administrated. After five cycles of the treatment, in September 2019, a PET-CT examination found a new emerging enlargement lymph node in the retroperitoneum and with an elevated SUV. In October 2019, after obtaining the patient's consent, we gave him PD-1 immune checkpoint treatment. And 9 cycles later, PET-CT showed that the enlargement lymph node found last time in the retroperitoneum had disappeared completely, with no other lesions were found. All the courses of treatment went through smoothly, and no severe toxicity happened. Immune checkpoint blockade is successful in R/R HIV-cHL, the toxicities are mild and accepted.
\end{abstract}

Keywords: Immune checkpoint inhibitor; human immunodeficiency virus; Hodgkin lymphoma (HL)

Submitted Jun 09, 2020. Accepted for publication Jul 15, 2020.

doi: $10.21037 /$ apm-20-1333

View this article at: http://dx.doi.org/10.21037/apm-20-1333

\section{Introduction}

Although classical Hodgkin lymphoma (cHL) is usually curable with the traditional treatments such as chemotherapy and radiation, but unfortunately, a few patients will eventually develop to relapsed or refractory cases. There many ways to deal with this situation including second-line chemotherapy, autologous stem cell transplantation, monoclonal anti-CD30 antibody and immune checkpoint inhibitor such as anti-PD-1 drug, etc. Nowadays, anti-PD-1 drug isn't only used in cHL, but also widely applicated in many solid cancers such as non-small cell lung cancer. Sintilimab (Innovent Biologics, Suzhou, China) is a highly selective, humanized, monoclonal antibody that blocks the interaction between PD-1 and its 15 ligands. As an anti-PD-1 drug, Sintilimab has improved 16 the outcome of patients with relapsed/refractory classical 17 Hodgkin lymphoma (R/R cHL) in a multicentre, single-arm 18 phase II trial, but to R/R HIV-cHL patients, no any case treated with Sintilimab was reported although a few cases were reported with the similar drugs such as Nivolumab.

We present the following case in accordance with the CARE reporting checklist (available at http://dx.doi. org/10.21037/apm-20-1333).

\section{Case presentation}

A 31-year-old Chinese man with R/R HIV-cHL has 


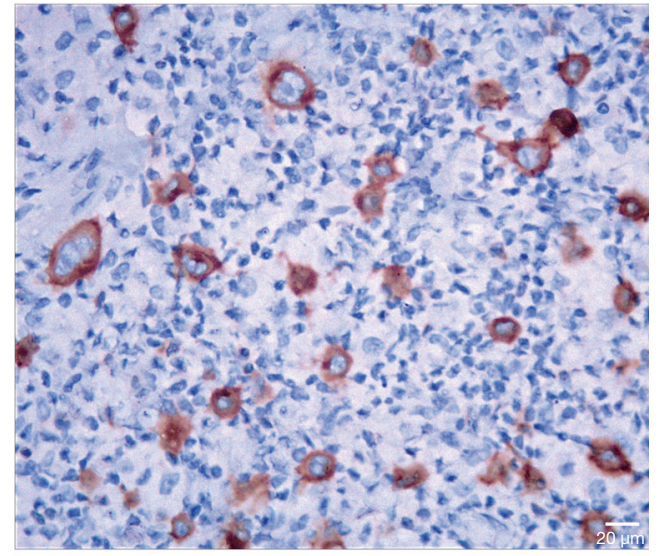

CD30 $\quad \mathrm{IH} 400 \times$

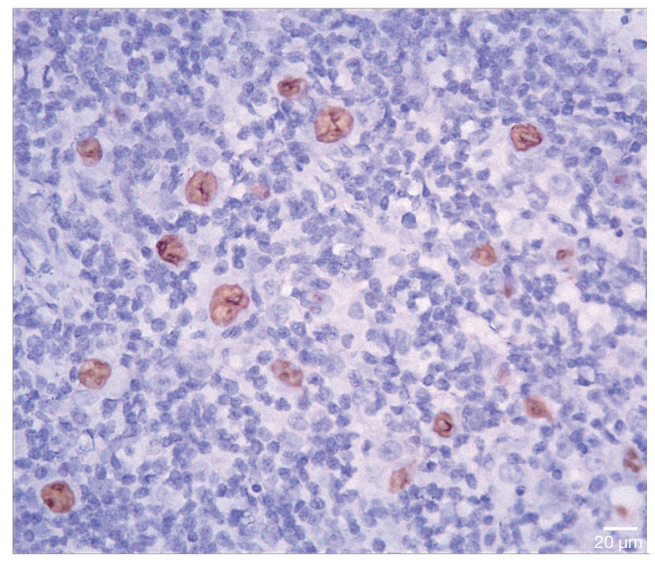

EBER IH 400x

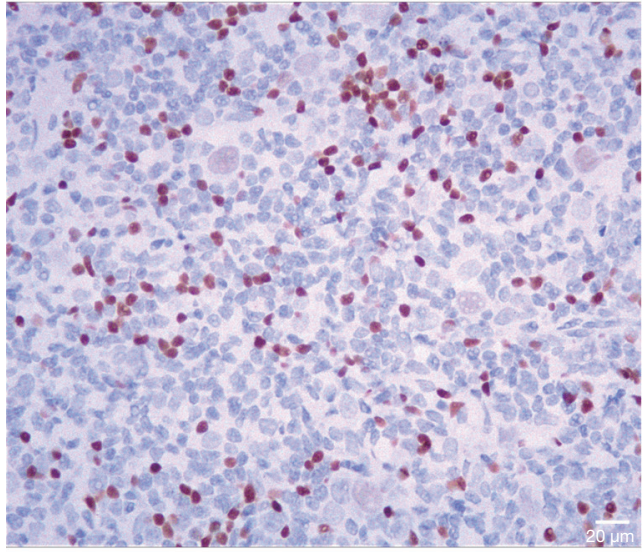

PAX-5 IH 400x

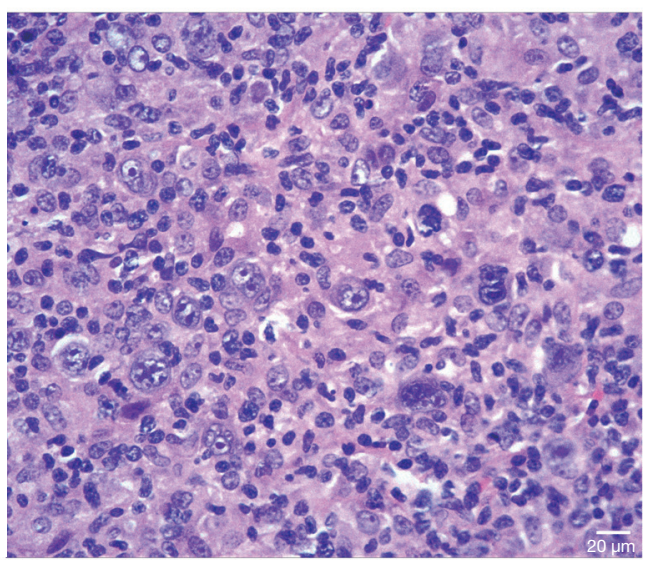

RS cell H\&E 1,000x

Figure 1 Pathologic analysis. H\&E, hematoxylin and eosin; IH, immunohistochemical staining; RS cell, Reed-Sternberg cell.

successfully reached complete remission after nine cycles of the PD-1 blockade Sintilimab Injection treatment.

The patient found himself enlarged lymph nodes in both side cervix in April 2018, without fever, night sweat, and weight loss. He was diagnosed with AIDS in March 2015 and has taken oral anti-retrovirus treatment regularly since then, and his status was under control with undetectable viral load and AIDS-related symptoms. After biopsy and pathological examination in the local hospital, the patient was diagnosed as lymphocyte-rich classical Hodgkin lymphoma (LRCHL), the HE shows: CD3-, CD30+, CD15-, PAX-5+ (weak), CD20+, EBER+, Ki67 30\%, CD45-, CD79a-, MUM-1+, BCL2- (Figure 1). PET-CT examinations showed multiple enlarged lymph nodes in the bilateral neck, submandibular, clavicle, double axilla, mediastinum, right hila, abdominal cavity, retroperitoneum, and bilateral pelvic wall near iliac vessels and groin. Further, an elevated SUV and Splenomegaly with elevated SUV were observed. Other laboratory checks showed albumin $\quad 47$ $38.75 \mathrm{~g} / \mathrm{dL}$; hemoglobin $10.9 \mathrm{~g} / \mathrm{dL}$; white blood cell count 48 $3,770.0 / \mathrm{mm}^{3}$; lymphocyte count $980 / \mathrm{mm}^{3}$. Finally, this 49 patient was diagnosed as HIV-LRCHL $\mathrm{III}_{\mathrm{E}+\mathrm{s}}$.

From May 2018, we began to give the patient 51 chemotherapy, he received the combination of ABVD 52 (doxorubicin, bleomycin, vinblastine, and dacarbazine) 53 regime for six cycles and prophylactic intrathecal (IT) 54 treatment(methotrexate $15 \mathrm{mg}$ every 4 weeks, for total $4 \quad 55$ times), all the courses went well, and no more than II grade 56 toxicities happened. In January 2019, the patient finished 57 the treatment and received a PET-CT check again, the 58 results showed the sizes of all the enlarged lymph nodes 59 reduced to normal, and Deauville scored less than 3, except 60 residual lesions in bilateral pelvic walls near iliac vessels and 61 groin with elevated SUV. All the residual lesions above were 62 treated with radical radiation, and the treatment went well. $\quad 63$

In April 2019, we evaluated efficacy six weeks after 64 

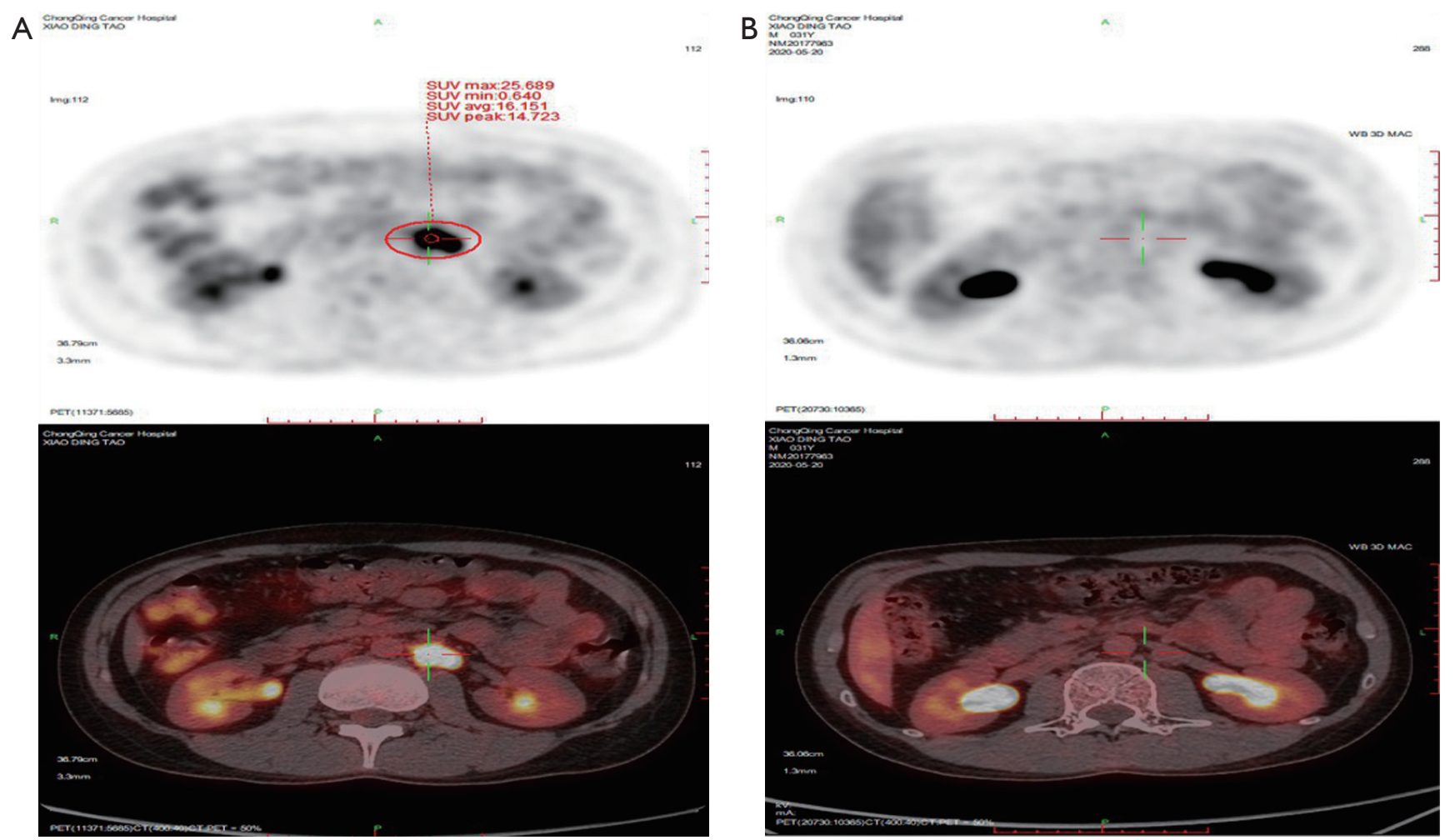

Figure 2 The change of the lesion before and after treatment. (A) The size and SUV of lymph node in the retroperitoneum by PET-CT examination before treatment. (B) PET-CT shows the lymph node in the retroperitoneum disappeared completely after Immune checkpoint inhibitor treatment.

radiotherapy. The PET-CT shows an enlarged lymph node with an elevated SUV in the left hila. The patient refused an auto stem cell transplant. Then we gave the patient the second line GDP (gemcitabine, dexamethasone, and cisplatin) regime chemotherapy. Unfortunately, after five cycles of treatment, in September 2019, PET-CT examination found new emerging enlargement lymph node in the retroperitoneum and with elevated SUV. However, other lesions disappeared (Figure 2A).

In October 2019, the patient aggressed to PD-1 immune checkpoint treatment. The Sintilimab Injection has prescribed $200 \mathrm{mg}$ ever 3 weeks, all the courses of treatment went through smoothly and no III-IV grade toxicity, after 9 cycles of treatment, we evaluated the effect of treatment, PET-CT showed that the new emerging enlargement lymph node found last time in the retroperitoneum has disappeared completely (Figure 2B).

All procedures performed in studies involving human participants were in accordance with the ethical standards of the institutional and/or national research committee(s) and with the Helsinki Declaration (as revised in 2013).
Written informed consent was obtained from the patient for publication of this study and any accompanying images.

\section{Discussion}

Hodgkin lymphoma (HL) is an uncommon malignancy involving lymph nodes and the lymphatic system and is divided into two main types, according to the WHO classification: $\mathrm{cHL}$ and nodular lymphocyte-predominant Hodgkin lymphoma (NLPHL). Furthermore, cHL includes four subtypes: nodular sclerosis cHL; mixed cellularity cHL; lymphocyte-depleted cHL; and lymphocyte-rich cHL (1)

Studies have shown that human immunodeficiency virus infection increases the risk of tumorigenesis and that patients with acquired immunodeficiency syndrome are 100 times more likely to develop lymphoma than the general population $(2,3)$. The most common subtypes of NHL in people living with HIV are diffuse large B-cell lymphoma (DLBCL), Burkitt lymphoma (BL), and primary central nervous system lymphoma (PCNSL), and the incidences of HL are also elevated in HIV positive patients but is 
much less common than BL or DLBCL (4). Although HIV-positive HL is not AIDS-defined cancer, the risk of HL in HIV-positive individuals is 10-20 times that of the general population (5). But the reason for Hodgkin lymphomagenesis in the HIV infected population is not clear, some studies show EBV could be detected in almost all HIV-HL patients, comparing that in HIV-negative ones (6). As we know, the 9p24.1 locus contains the genes encoding programmed cell death 1 ligand 1 and 2 (PD-L1 and PD-L2), and JAK2; lymphoma-associated aberrations in this locus result in increased expression of these proteins, and the EBV-latent membrane protein (LMP1) may induce PD-L1 expression via the AP-1 and JAK-STAT pathways in cHL cells with diploid 9p24.1 $(7,8)$.

Compared to the HIV-negative patients, HIV patients with lymphoma tend to have more B-symptoms at presentation, greater extra-nodal involvement, increased likelihood of bone marrow disease, and poorer performance status. The histology types of mixed cellularity or lymphocyte-depleted in classical HL companied with Human immunodeficiency virus are more common, and the HIV-cHL is almost universally Epstein-Barr virus-positive (6). In the pre-combined antiretroviral therapy (cART) era, HIV-cHL prognosis was dismal due to the aggressiveness of the lymphoma and the poor prognosis of the HIV infection (9). With the widespread use of antiretroviral therapy, the overall incidence of HIV-associated Kaposi sarcoma, primary central nervous system lymphoma, and diffuse large B-cell lymphoma have been decreasing rapidly, but the incidence of HIV-cHL has not significantly decreased (10). With CARTs advent, although the incidence of CHL has increased, the response to treatment and long-term survival has also improved (11). Some studies have proved that HIVpositive patients with $\mathrm{cHL}$ had more aggressive baseline features. However, there were no differences in response rate or survival between HIV-positive and HIV-negative patients, not significantly associated with higher mortality in HIV-positive patients $(12,13)$.

Even the advancement of HIV-cHL prognosis, there are still a few patients that can get complete remission and eventually come into R/R HIV-cHL, so the salvage treatments are challenging. Studies are finding a high proportion of cHL tumor harboring cells expressing PD-1 ligands, which has provided the rationale for clinical trials of inhibitors of this immune checkpoint (7).

There many ways to deal with R/R HIV-cHL including second-line chemotherapy, autologous stem cell transplantation, monoclonal anti-CD30 antibody and 155 immune checkpoint inhibitor, etc. Immune checkpoint 156 blockade emerged as an essential treatment option for 157 patients with relapsed or refractory HL in 2015 after 158 a pivotal single-arm multicenter trial of nivolumab 159 monotherapy demonstrated an impressive objective 160 response rate of $87 \%$ with mild toxicities $(14,15)$. Another 161 prospective treatment is CD30-specific CAR-T, but there 162 are only a few clinical trials having been reported. In a 163 phase I clinical trial with R/R HL and Anaplastic Large 164 Cell Lymphoma (ALCL) showed that CD30-specific 165 CAR-Ts are safe, indicating that further assessment of 166 this therapy is warranted (16). Nowadays, there is no any 167 evidences whether anti-PD-1 drugs is useful for HIV or 168 not, some experts think anti-PD-1 therapy holding promise 169 as adjunctive therapy for chronic infectious diseases such as 170 $\mathrm{TB}$ and HIV, but need to be tested in randomized clinical 171 trials $(17,18)$. However, to HIV-cHL, only three cases 172 had been reported with the treatment of PD-1 immune 173 checkpoint, all the three cases had positive outcomes, and 174 one of them reached complete remission (CR) $(6,15,19) . \quad 175$ In our case, the young patient had experienced two 176 regimes of chemotherapy and radiation for 17 months and 177 finally progressed. After we gave him Sintilimab Injection 178 treatment for 9 cycles, he reached complete remission, with 179 our knowledge, this is first reported in Asia and the second 180 CR case in the world.

Sintilimab is effective in R/R HIV-cHL, and the toxicities are accepted, further prospective clinical trials to verify are valuable.

The patient now has no any symptoms, and can do some housework now. He is willing to continue this treatment.

\section{Acknowledgments}

Funding: Sponsored by Natural Science Foundation of Chongqing, China (cstc2019jcyj-msxmX0793).

\section{Footnote}

Reporting Checklist: The authors have completed the CARE reporting checklist. Available at http://dx.doi.org/10.21037/ apm-20-1333

Conflicts of Interest: All authors have completed the ICMJE uniform disclosure form (available at http://dx.doi. org/10.21037/apm-20-1333). The authors report grants from Natural Science Foundation of Chongqing, China, 
during the conduct of the study.

Ethical Statement: The authors are accountable for all aspects of the work in ensuring that questions related to the accuracy or integrity of any part of the work are appropriately investigated and resolved. All procedures performed in studies involving human participants were in accordance with the ethical standards of the institutional and/or national research committee(s) and with the Helsinki Declaration (as revised in 2013). Written informed consent was obtained from the patient for publication of this study and any accompanying images.

Open Access Statement: This is an Open Access article distributed in accordance with the Creative Commons Attribution-NonCommercial-NoDerivs 4.0 International License (CC BY-NC-ND 4.0), which permits the noncommercial replication and distribution of the article with the strict proviso that no changes or edits are made and the original work is properly cited (including links to both the formal publication through the relevant DOI and the license). See: https://creativecommons.org/licenses/by-nc-nd/4.0/.

\section{References}

1. Sabattini E, Bacci F, Sagramoso C, et al. WHO classification of tumours of haematopoietic and lymphoid tissues in 2008: an overview. Pathologica 2010;102:83-7.

2. Goedert JJ. The epidemiology of acquired immunodeficiency syndrome malignancies. Semin Oncol 2000;27:390-401.

3. Beral V, Peterman T, Berkelman R, et al. AIDS-associated non-Hodgkin lymphoma. Lancet 1991;337:805-9.

4. Gopal S, Patel MR, Yanik EL, et al. Temporal trends in presentation and survival for HIV-associated lymphoma in the antiretroviral therapy era. J Natl Cancer Inst 2013;105:1221-9.

5. Castillo JJ, Bower M, Brühlmann J, et al. Prognostic factors for advanced-stage human immunodeficiency virus-associated classical Hodgkin lymphoma treated with doxorubicin, bleomycin, vinblastine, and dacarbazine plus combined antiretroviral therapy: a multi-institutional retrospective study. Cancer 2015;121:423-31.

6. Thompson LD, Fisher SI, Chu WS, et al. HIVassociated Hodgkin lymphoma: a clinicopathologic and immunophenotypic study of 45 cases. Am J Clin Pathol 2004;121:727-38.
7. Goodman A, Patel SP, Kurzrock R. PD-1-PD-L1 251 immune-checkpoint blockade in B-cell lymphomas. Nat 252 Rev Clin Oncol 2017;14:203-20.

8. Green MR, Rodig S, Juszczynski P, et al. Constitutive AP-1 activity and EBV infection induce PD-L1 in Hodgkin lymphomas and posttransplant lymphoproliferative disorders: implications for targeted therapy. Clin Cancer Res 2012;18:1611-8.

9. Levine AM, Li P, Cheung T, et al. Chemotherapy consisting of doxorubicin, bleomycin, vinblastine, and dacarbazine with granulocyte-colony-stimulating factor in HIV-infected patients with newly diagnosed Hodgkin's disease: a prospective, multi-institutional AIDS clinical trials group study (ACTG 149). J Acquir Immune Defic Syndr 2000;24:444-50.

10. Hernández-Ramírez RU, Shiels MS, Dubrow R, et al. Cancer risk in HIV-infected people in the USA from 1996 to 2012: a population-based, registry-linkage study. Lancet HIV 2017;4:e495-504.

11. Besson C, Lancar R, Prevot S, et al. High Risk Features Contrast With Favorable Outcomes in HIV-associated Hodgkin Lymphoma in the Modern cART Era, ANRS CO16 LYMPHOVIR Cohort. Clin Infect Dis 2015;61:1469-75.

12. Sorigué M, García O, Tapia G, et al. HIV-infection has no prognostic impact on advanced-stage Hodgkin lymphoma. AIDS 2017;31:1445-9.

13. Olszewski AJ, Castillo JJ. Outcomes of HIV-associated Hodgkin lymphoma in the era of antiretroviral therapy. AIDS 2016;30:787-96.

14. Ansell SM, Lesokhin AM, Borrello I, et al. PD-1 blockade with nivolumab in relapsed or refractory Hodgkin's lymphoma. N Engl J Med 2015;372:311-9.

15. Chang E, Rivero G, Patel NR, et al. HIV-related Refractory Hodgkin Lymphoma: A Case Report of Complete Response to Nivolumab. Clin Lymphoma Myeloma Leuk 2018;18:e143-6.

16. Ramos CA, Ballard B, Zhang H, et al. Clinical and immunological responses after CD30-specific chimeric antigen receptor-redirected lymphocytes. J Clin Invest. 2017;127:3462-71.

17. Filaci G, Fenoglio D, Taramasso L, et al. Rationale for an Association Between PD1 Checkpoint Inhibition and Therapeutic Vaccination Against HIV. Front Immunol 2018;9:2447.

18. Rao M, Valentini D, Dodoo E, et al. Anti-PD-1/PD-L1 therapy for infectious diseases: learning from the cancer 
paradigm. Int J Infect Dis 2017;56:221-8.

19. Sandoval-Sus JD, Mogollon-Duffo F, Patel A, et al. Nivolumab as salvage treatment in a patient with HIVrelated relapsed/refractory Hodgkin lymphoma and liver failure with encephalopathy. J Immunother Cancer 2017;5:49.

(English Language Editor: J. Chapnick)

Cite this article as: Shi Y, Li Q, Zhang W, Nan Y, Yang T, Liang X, Xiao C, Guo B, Xiang Y. Sintilimab as salvage treatment in an HIV patient with relapsed/refractory Hodgkin: a case report. Ann Palliat Med 2020;9(4):2414-2419. doi: 10.21037/apm-20-1333 